\title{
Resistance in gastrointestinal stromal tumors
}

\author{
Andreas Seeber · Alexander Perathoner - Florian Kocher
}

Received: 8 January 2019 / Accepted: 26 March 2019 / Published online: 18 April 2019

(C) The Author(s) 2019

\begin{abstract}
Summary The approval of imatinib for the treatment of patients with gastrointestinal stromal tumor has (GIST) revolutionized the treatment in the adjuvant setting and metastatic disease. Imatinib is an inhibitor of the receptor tyrosine kinases KIT and platelet-derived growth factor receptor (PDGFR), which are constitutively activated in most cases of GIST. Even though substantial survival improvements have been observed with imatinib, primary or secondary resistance to imatinib represents a major challenge in the treatment of GIST. This short review focusses on treatment strategies to overcome resistance and provides an overview of promising new agents currently evaluated for imatinib-refractory disease.
\end{abstract}

Keywords GIST $\cdot$ Resistance $\cdot$ Imatinib $\cdot \mathrm{KIT} \cdot \mathrm{PDGFR} \alpha$

\section{Introduction}

Gastrointestinal stromal tumors (GIST) are a distinct subgroup of mesenchymal neoplasms. Although it is a very rare tumor entity with an annual incidence of approximately 1:100,000, it represents the most common non-epithelial type of cancer in the gastrointestinal tract $[1,2]$. The only curative treatment for GIST is radical surgery at the right time. Adjuvant therapy is indicated in cases with a high risk of recurrence following the risk classification of Miettinen and Lasota [3]. The liver is the most common metastatic

\footnotetext{
A. Seeber · F. Kocher $(\bowtie)$

Department of Internal Medicine V: Haematology and Oncology, Medical University of Innsbruck, Anichstraße 35, 6020 Innsbruck, Austria florian.kocher@i-med.ac.at

\section{A. Perathoner}

Department of Visceral, Transplant, and Thoracic Surgery, Medical University of Innsbruck, Innsbruck, Austria
}

site in patients with GIST, approximately $10 \%$ of patients present with synchronous liver metastases at the time of diagnosis [4]. For a long time, survival in metastatic disease remained poor due to the low efficacy of chemo- and radiotherapy in GIST. However, treatment of metastatic GIST has changed substantially during the past two decades. The identification of the nearly unique expression of the CD117 antigen in GIST, which is a subunit of the KIT receptor encoded by the KIT proto-oncogene, led to a deeper understanding of the pathophysiology of GIST $[5,6]$. Activated KIT mutations induce activation of downstream pathways (i.e., JAK/STAT or RAS/MAP) with induction of tumorigenesis and proliferation [7]. It is estimated that approximately $80 \%$ of all GISTs harbor a mutation in KIT [8].

Imatinib, a tyrosine kinase inhibitor (TKI), has dramatically changed the treatment of chronic myeloid leukemia (CML). Imatinib blocks the activated abl kinase, which is responsible for proliferation in CML [9]. It was only a matter of time before it was noticed that abl, KIT, and platelet-derived growth factor receptor-alpha (PDGFR $\alpha$ ) show a similar chemical structure and therefore provide a biologic rationale to evaluate imatinib in patients with GIST [10]. In the first clinical trial conducted by Demetri and colleagues, imatinib was tested on 147 patients with nonresectable or metastatic GIST. In this study, radiographic response within 6 months was observed in 54\% of patients [11]. Long-term results confirmed the efficacy of imatinib and yielded a 5-year overall survival (OS) of nearly 50\% (median OS: 57 months) and a median progression-free survival (PFS) of 24 months [12]. A further analysis revealed that outcome in GIST patients undergoing treatment with imatinib depends on specific KIT mutations. It was observed that exon 11 alterations are associated with better OS and PFS compared with exon 9 mutations or wild-type (WT) KIT 
[13]. Today, guidelines suggest treatment with imatinib in advanced GIST patients harboring a sensitive KIT mutation (i.e., exon 11, exon 9; [14]). Owing to an inferior outcome in patients with exon 9 mutations, an increased dose of imatinib (800 mg daily) is suggested in this subset of patients. The rationale for increased dosing in GIST with exon 9 mutations is based on a meta-analysis comparing different doses of imatinib in GIST. According to this analysis, imatinib $800 \mathrm{mg}$ was associated with improved PFS compared with conventional dosing (400 mg daily) in patients with exon 9 mutation [15].

\section{Primary and secondary resistance to imatinib}

Although imatinib is the mainstay of therapy in metastatic GIST, there are subgroups of patients showing primary resistance to imatinib. In approximately $5-7 \%$ of GISTs, no KIT alterations but instead PDGFR $\alpha$ alterations are detected. The PDGFR $\alpha$ D824V mutation is the most common alteration associated with primary imatinib resistance. According to a study evaluating outcome in GIST patients harboring PDGFR $\alpha$ mutations, no patient with PDGFR $\alpha$ D824V showed response to imatinib [16]. Another subgroup of GISTs, which only occasionally occurs in adults, is characterized by the lack of KIT and PDGFR $\alpha$ mutations. This distinct molecular entity is therefore classified as WT-GIST. Most of these tumors show mutations in the SDHX (succinyl dehydrogenase) gene. In an observational study of WT-GIST, response was only seen in one of 49 patients treated with imatinib [17].

The most common acquired resistance mechanisms are induced by secondary KIT mutations. These secondary mutations are usually located in the ATPbinding domains of KIT and PDGFR $\alpha$, thereby hindering the binding of imatinib to the target structure [18].

\section{Strategies to overcome resistance}

In patients with disease progression on imatinib treatment, dose escalation might be considered. In a subgroup analysis of a randomized dose-finding study, stable disease (SD) was observed in $27 \%$ of patients who crossed over from the $400 \mathrm{mg}$ to the $800 \mathrm{mg}$ imatinib treatment arm because of progressive disease (PD). Partial response (PR) was seen in $2 \%$ of patients [19]. In an American dose-finding study, comparable results were observed with SD and objective response (ORR) in 28 and 3\%, respectively [20].

To date, two other agents have been approved for patients with GIST who have disease progression on imatinib first-line therapy: sunitinib and regorafenib. Sunitinib is an oral multikinase inhibitor targeting several kinases, including PDGFR $\alpha$, VEGFR, as well as KIT. Sunitinib is suggested as standard secondline therapy [14]. A pivotal phase III trial met its primary endpoint by showing prolonged PFS (6.3 vs. 1.5 months) and increased time-to-progression (TTP; 27 vs. 6 weeks) in patients treated with sunitinib compared with placebo. A prolongation of OS was not observed owing to the pre-specified cross-over study design [21]. Interestingly, clinical activity of sunitinib in GIST seems to depend on the mutational status. A phase I/II trial with patients resistant or intolerant to imatinib and subsequent treatment with sunitinib revealed that PFS rates in the KIT exon 9 mutations and WT-GIST groups were significantly longer than in patients harboring primary exon 11 mutations [22]. Comparable mechanisms as in secondary imatinib-refractory disease are suspected to induce sunitinib resistance [23].

For GIST patients showing a PD on imatinib as well as sunitinib, regorafenib is a further treatment option. This oral multikinase inhibitor targets KIT, PDGFR, FGRFR, BRAF, RET, VEGF1-3, and TIE2 [24]. In a phase II trial investigating regorafenib after imatinib and sunitinib disease progression, a clinical benefit rate (CBR) of $79 \%$ and a median PFS of 10.0 months were observed [25]. The subsequent phase III GRID trial, evaluating regorafenib versus placebo, revealed a prolongation of PFS of approximately 4 months in the regorafenib arm (4.8 vs. 0.9 months, $p<0.001$ ).

Other oral TKIs have also been evaluated for GIST patients. Sorafenib, a multikinase inhibitor targeting KIT, VEGFR, and PDGFR, was evaluated in a small phase II trial in patients with imatinib or imatinib/ sunitinib-refractory disease. In this trial, a disease control rate (DCR) of $68 \%$ and a median PFS of 5.2 months were reported [26]. Ponatinib was tested in patients with GIST refractory to approved agents. Patients were stratified according to the presence or absence of exon 11 mutations. The CBR of $>16$ weeks in patients without exon 11 mutation was $55 \%$ whereas in patients with primary exon 11 mutations the CBR was $22 \%$ [27]. In a phase III trial, nilotinib, a secondgeneration TKI, was investigated in GIST patients after imatinib and sunitinib failure. The median PFS, assessed by local investigators, was significantly longer in the nilotinib arm compared with patients receiving best supportive care (119 vs. 70 days, $p=0.0007$ ). However, according to central radiologic review, no PFS benefit was observed [28]. The PAZOGIST trial evaluated the activity of pazopanib in GIST after imatinib/sunitinib progression. The 4-month PFS was significantly improved in patients receiving pazopanib compared with best supportive care (45\% vs. $18 \%, p=0.029$; [29]). Dasatinib has shown promising activity in GIST including patients with WT-GIST and PDGFR $\alpha$ mutations. According to a single-arm trial, in imatinib-refractory patients undergoing treatment with dasatinib, a 6-month PFS rate of $29 \%$ and ORR of $25 \%$ was observed [30]. 


\section{Potential new drugs}

Besides the aforementioned TKIs, other agents are currently being investigated for metastatic GIST. In vitro studies indicate promising activity of crenolanib, a receptor tyrosine kinase inhibitor, in GIST harboring a PDGFR $\alpha$ D824V mutation [31]. Crenolanib is currently evaluated in a phase III trial in GIST patients with this particular gene alteration (NCT02847429). A phase II trial revealed that olaratumab, a monoclonal antibody targeting PDGFR $\alpha$, only shows modest activity in GIST. However, in patients harboring a PDGFR $\alpha$ D824V mutation, olaratumab treatment might lead to a longer DCR [32]. Only recently, the first results of checkpoint inhibition in metastatic GIST were published. In a phase II trial, the CBRs in patients receiving nivolumab (anti-PD-1 antibody) or nivolumab/ipilimumab (anti-CTLA-4 antibody) were 43 and $40 \%$, respectively. Another study, investigating pembrolizumab (anti-PD-L1 antibody) in combination with epacadostat (IDO-inhibitor), is currently recruiting. Study completion is planned in 2019.

Avapritinib, a highly selective inhibitor of mutated KIT and PDGFR $\alpha$, has shown significant activity in GIST. In the phase I NAVIGATOR trial in pretreated GIST, avapritinib demonstrated encouraging activity with an ORR of $84 \%$ (CR 9\%, PR 75\%). The CBR at 4 months was $96 \%$ [33]. The ongoing phase III VOYAGER study is currently evaluating avapritinib vs. regorafenib in pretreated GIST patients (NCT03465722). DCC-2618, a KIT/PDGFR $\alpha$ switch-control inhibitor, represents another potent agent. According to the first results of a phase I trial, an ORR of $14 \%$ and a DCR at 3 months of $70 \%$ were achieved in GIST patients undergoing treatment with DCC-2618 [34]. Based on these results, a phase III trial will evaluate the agent in the fourth-line setting (NCT03353753). Furthermore, the oral multikinase inhibitor cabozantinib is currently tested within an EORTC phase II trial (NCT-02216578) in patients with disease progression on imatinib and sunitinib therapies.

\section{Conclusion}

The advances in oncologic research and the understanding of fundamental molecular cancer pathways have led to highly effective targeted treatments in patients with GIST in the past 10 years. The mechanisms of TKI resistance represent a major treatment challenge. Currently, several new potentially active agents are being evaluated in different clinical trials to overcome these resistances. The results of these studies are awaited in the next few years. It remains to be seen which therapies will enrich the treatment arsenal in GIST.

\section{Take-home message}

Imatinib is a mainstay of therapy for GIST. Resistance to imatinib remains a major challenge. Second- and thirdline agents are in clinical use and several further agents are currently evaluated in clinical trials to overcome imatinib resistance and to improve survival.

Funding Open access funding provided by University of Innsbruck and Medical University of Innsbruck.

Conflict of interest A. Seeber, A. Perathoner, and F. Kocher declare that they have no competing interests.

Open Access This article is distributed under the terms of the Creative Commons Attribution 4.0 International License (http://creativecommons.org/licenses/by/4.0/), which permits unrestricted use, distribution, and reproduction in any medium, provided you give appropriate credit to the original author(s) and the source, provide a link to the Creative Commons license, and indicate if changes were made.

\section{References}

1. Rubin BP,HeinrichMC,CorlessCL.Gastrointestinal stromal tumour. Lancet. 2007;369(9574):1731-41.

2. Ma GL, Murphy JD, Martinez ME, SicklickJK. Epidemiology of gastrointestinal stromal tumors in the era of histology codes: results of a population-based study. Cancer Epidemiol Biomarkers Prev. 2015;24(1):298-302.

3. Miettinen M, Lasota J. Gastrointestinal stromal tumors: pathology and prognosis at different sites. Semin Diagn Pathol. 2006;23(2):70-83.

4. Gaitanidis A, Alevizakos M, Tsaroucha A, Simopoulos C, Pitiakoudis M. Incidence and predictors of synchronous liver metastases in patients with gastrointestinal stromal tumors (GISTs). Am J Surg. 2018;216(3):492-7.

5. Hirota S, Isozaki K, Moriyama Y, Hashimoto K, Nishida $\mathrm{T}$, Ishiguro S, et al. Gain-of-function mutations of c-kit in human gastrointestinal stromal tumors. Science. 1998;279(5350):577-80.

6. Besmer P, Murphy JE, George PC, Qiu FH, Bergold PJ, Lederman L, et al. A new acute transforming feline retrovirus and relationship of its oncogene v-kit with the protein kinase gene family. Nature. 1986;320(6061):415-21.

7. Lennartsson J, Ronnstrand L. Stem cell factor receptor/cKit: from basic science to clinical implications. Physiol Rev. 2012;92(4):1619-49.

8. Emile JF, Theou N, Tabone S, Cortez A, Terrier P, Chaumette MT, et al. Clinicopathologic, phenotypic, and genotypic characteristics of gastrointestinal mesenchymal tumors. Clin Gastroenterol Hepatol. 2004;2(7):597-605.

9. Druker BJ, Talpaz M, Resta DJ, Peng B, Buchdunger E, Ford JM, et al. Efficacy and safety of a specific inhibitor of the BCR-ABL tyrosine kinase in chronic myeloid leukemia. NEngl J Med. 2001;344(14):1031-7.

10. Heinrich MC, Griffith DJ, Druker BJ, Wait CL, Ott KA, Zigler AJ. Inhibition of c-kit receptor tyrosine kinase activity by STI 571, a selective tyrosine kinase inhibitor. Blood. 2000;96(3):925-32.

11. Demetri GD, von Mehren M, Blanke CD, Van den Abbeele $\mathrm{AD}$, Eisenberg B, Roberts PJ, et al. Efficacy and safety of imatinib mesylate in advanced gastrointestinal stromal tumors. NEngl J Med. 2002;347(7):472-80.

12. Blanke CD, Demetri GD, von Mehren M, Heinrich MC, Eisenberg B, Fletcher JA, et al. Long-term results from 
a randomized phase II trial of standard- versus higherdose imatinib mesylate for patients with unresectable or metastatic gastrointestinal stromal tumors expressing KIT. JClin Oncol. 2008;26(4):620-5.

13. PatrikidouA, DomontJ,ChabaudS, Ray-Coquard I, Coindre JM, Bui-Nguyen B, et al. Long-term outcome of molecular subgroups of GIST patients treated with standard-dose imatinib in the BFR14 trial of the French Sarcoma Group. Eur JCancer. 1990;52:173-80.

14. Casali PG, Abecassis N, Aro HT, Bauer S, Biagini R, Bielack S, et al. Gastrointestinal stromal tumours: ESMO-EURACAN Clinical Practice Guidelines for diagnosis, treatment and follow-up. Ann Oncol. 2018;29(Supplement_4):iv267.

15. Gastrointestinal Stromal Tumor Meta-Analysis Group (MetaGIST). Comparison of two doses of imatinib for the treatment of unresectable or metastatic gastrointestinal stromal tumors: a meta-analysis of 1,640 patients. J Clin Oncol. 2010;28(7):1247-53.

16. Cassier PA, Fumagalli E, Rutkowski P, Schoffski P, Van Glabbeke M, Debiec-Rychter M, et al. Outcome of patients with platelet-derived growth factor receptor alphamutated gastrointestinal stromal tumors in the tyrosine kinase inhibitor era. Clin Cancer Res. 2012;18(16):4458-64.

17. Boikos SA, Pappo AS, Killian JK, LaQuaglia MP, Weldon CB, George S, et al. Molecular subtypes of KIT/PDGFRA wildtype gastrointestinal stromal tumors: a report from the national institutes of health gastrointestinal stromal tumor clinic. JAMA Oncol. 2016;2(7):922-8.

18. Wardelmann E, Merkelbach-Bruse S, Pauls K, Thomas N, Schildhaus HU, Heinicke T, et al. Polyclonal evolution of multiple secondary KIT mutations in gastrointestinal stromal tumors under treatment with imatinib mesylate. Clin Cancer Res. 2006;12(6):1743-9.

19. Zalcberg JR, Verweij J, Casali PG, Le Cesne A, Reichardt P, Blay JY, et al. Outcome of patients with advanced gastrointestinal stromal tumours crossing over to a daily imatinib dose of $800 \mathrm{mg}$ after progression on $400 \mathrm{mg}$. Eur J Cancer. 1990;41(12):1751-7.

20. BlankeCD, Rankin C, Demetri GD, Ryan CW, von Mehren M, Benjamin RS, et al. Phase III randomized, intergroup trial assessing imatinib mesylate at two dose levels in patients with unresectable or metastatic gastrointestinal stromal tumors expressing the kit receptor tyrosine kinase: S0033. JClin Oncol. 2008;26(4):626-32.

21. Demetri GD, van Oosterom AT, Garrett CR, Blackstein ME, Shah MH, Verweij J, et al. Efficacy and safety of sunitinib in patients with advanced gastrointestinal stromal tumour after failure of imatinib: a randomised controlled trial. Lancet. 2006;368(9544):1329-38.

22. Heinrich MC, Maki RG, Corless CL, Antonescu CR, Harlow A, Griffith D, et al. Primary and secondary kinase genotypes correlate with the biological and clinical activity of sunitinib in imatinib-resistant gastrointestinal stromal tumor. J Clin Oncol. 2008;26(33):5352-9.

23. Guo T, Hajdu M, Agaram NP, Shinoda H, Veach D, Clarkson $\mathrm{BD}$, et al. Mechanisms of sunitinib resistance in gastrointestinal stromal tumors harboring KITAY502-3ins mutation: an in vitro mutagenesis screen for drug resistance. Clin Cancer Res. 2009;15(22):6862-70.

24. Wilhelm SM, Dumas J, Adnane L, Lynch M, Carter CA, Schutz G, et al. Regorafenib (BAY 73-4506): a new oral multikinase inhibitor of angiogenic, stromal and oncogenic receptor tyrosine kinases with potent preclinical antitumor activity. Int J Cancer. 2011;129(1):245-55.

25. GeorgeS, Wang Q, Heinrich MC, Corless CL, Zhu M, Butrynski JE, et al. Efficacy and safety of regorafenib in patients with metastatic and/or unresectable GI stromal tumor after failure ofimatinib and sunitinib: a multicenter phase II trial. JClin Oncol. 2012;30(19):2401-7.

26. Campbell NP, Wroblewski K, Maki RG, D’Adamo DR, Chow WA, Gandara DR, et al. Final results of a University of Chicago phase II consortium trial of sorafenib (SOR) in patients (pts) with imatinib (IM) - and sunitinib (SU)-resistant (RES) gastrointestinal stromal tumors (GIST). J Clin Oncol. 2011;29(4_suppl):4.

27. Heinrich MC, vonMehren M, Demetri GD, Fletcher JA, Sun J, Hodgson JG, et al. A phase 2 study of ponatinib in patients (pts) with advanced gastrointestinal stromal tumors (GIST) afterfailure of tyrosine kinaseinhibitor (TKI) therapy: Initial report. JClin Oncol. 2014;32(15_suppl):10506.

28. Reichardt P, Blay JY, Gelderblom H, Schlemmer M, Demetri GD, Bui-Nguyen B, et al. Phase III study of nilotinib versus best supportive care with or without a TKI in patients with gastrointestinal stromal tumors resistant to or intolerant of imatinib and sunitinib. Ann Oncol. 2012;23(7):1680-7.

29. Mir O, Cropet C, Toulmonde M, Cesne AL, Molimard M, Bompas E, et al. Pazopanib plus best supportive care versus best supportive care alone in advanced gastrointestinal stromal tumours resistant to imatinib and sunitinib (PAZOGIST): a randomised, multicentre, open-label phase 2 trial. Lancet. 2016;17(5):632-41.

30. Schuetze SM, Bolejack V, Thomas DG, von Mehren M, Patel S, Samuels B, et al. Association of dasatinib with progression-free survival among patients with advanced gastrointestinal stromal tumors resistant to Imatinib. JAMA. 2018;4(6):814-20.

31. Heinrich MC, Griffith D, McKinley A, Patterson J, Presnell A, Ramachandran A, et al. Crenolanib inhibits the drug-resistant PDGFRA D842V mutation associated with imatinibresistant gastrointestinal stromal tumors. Clin Cancer Res. 2012;18(16):4375-84.

32. Wagner AJ, Kindler H, Gelderblom H, Schoffski P, Bauer S, Hohenberger P, et al. A phase II study of a human antiPDGFRalpha monoclonal antibody (olaratumab, IMC3G3) in previously treated patients with metastatic gastrointestinal stromal tumors. Ann Oncol. 2017;28(3):541-6.

33. Heinrich M, von Mehren M, Jones RL, et al. Avapritinib is highly active and well-tolerated in patients (PTS) with advanced GIST driven by diverse variety of oncogenic mutations in KIT and PDGFRA. Annual Meeting of the Connective Tissue Oncology Society; 14.11.-17.11.; Rome. 2018.

34. GeorgeS, Heinrich M, ChiP, RazakA, von Mehren M, Gordon M, Ganjoo KN, Somaiah N, Trent JC, Rodon Ahnert J, Wolf J, Ruiz-Soto R, Rosen O, Janku F. Initial results of phase 1 study of DCC-2618, a broad-spectrum KIT and PDGFRa inhibitor, in patients (pts) with gastrointestinal stromal tumor. Ann Oncol. 2018;29(suppl_8):viii576-viii95.

Publisher's Note Springer Nature remains neutral with regard to jurisdictional claims in published maps and institutional affiliations.

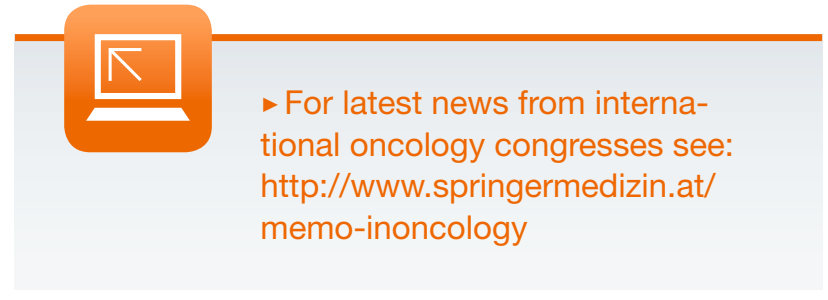

